\title{
Aleitamento materno e práticas alimentares de crianças menores de seis meses em Alfenas, Minas Gerais
}

\author{
Breastfeeding and feeding practices for infants under \\ six months of age from Alfenas, Minas Gerais, Brazil
}

RE S U M O

Cássia Irene Spinelli ARANTES

Maísa Muniz OLIVEIRA²

Thaís Cristina Rodrigues VIEIRA²

Luiz Alberto BEIJO3

Clícia Valim Côrtes GRADIM ${ }^{1}$

Sueli Leiko Takamatsu GOYATÁ ${ }^{1}$

\section{Objetivo}

Determinar a prevalência da amamentação e avaliar o consumo de outros alimentos em crianças menores de seis meses em Alfenas, Estado de Minas Gerais.

\section{Métodos}

Trata-se de estudo epidemiológico transversal, realizado por meio de inquérito domiciliar com uma amostra de 126 crianças nascidas em 2008. Os dados foram obtidos a partir de formulário com o recordatório de 24 horas da alimentação da criança e outras questões. Na análise dos dados, foi utilizado o Programa Epi Info, versão 6.04. Foi determinada a prevalência das diferentes categorias de amamentação (amamentação exclusiva, amamentação predominante, amamentação complementada e amamentação) segundo faixas etárias. O Teste de Fischer e o Teste qui-quadrado foram calculados na associação de variáveis com o aleitamento materno.

\section{Resultados}

A prevalência da amamentação exclusiva em crianças menores de seis meses foi de 37,3\%. A introdução de alimentos semissólidos ocorreu acentuadamente a partir do quinto mês de vida. No sexto mês, 50,7\% das crianças encontravam-se em amamentação complementada. As crianças menores de seis meses não amamentadas, comparadas com as amamentadas, apresentaram prevalências significativamente maiores quanto ao consumo de água, frutas e comida da família. Não foram encontradas diferenças significativas entre a amamentação exclusiva até o sexto mês e as variáveis maternas estudadas.

\footnotetext{
1 Universidade Federal de Alfenas, Departamento de Enfermagem. R. Gabriel Monteiro da Silva, 700, 37130-000, Alfenas, MG, Brasil. Correspondência para/Correspondence to: C.I.S. ARANTES. E-mail: <cassiaarantes@terra.com.br>.

2 Universidade Federal de Alfenas, Curso de Enfermagem. Alfenas, MG, Brasil.

${ }^{3}$ Universidade Federal de Alfenas, Departamento de Ciências Exatas. Alfenas, MG, Brasil.
} 
422 | C.I.S. ARANTES et al.

\section{Conclusão}

Apesar de vários resultados deste estudo apresentarem-se superiores aos de outros municípios, a prevalência do aleitamento materno em Alfenas ainda está distante da recomendação de que crianças recebam exclusivamente leite materno até os seis meses de idade.

Termos de indexação: Aleitamento materno. Leite humano. Nutrição do lactante. Prevalência.

\section{A B S T R A C T}

\section{Objective}

This study determined the prevalence of breastfeeding and evaluated the consumption of other foods by infants under six months of age from Alfenas, Minas Gerais.

\section{Methods}

This cross-sectional, epidemiological study included 126 children born in 2008. The infants' caregivers were interviewed at home using the $24 \mathrm{hrs}$ recall and other questions to collect information on the infants' diets. The software Epi Info version 6.04 was used to analyze the data. The prevalence of different breastfeeding categories was determined (exclusive breastfeeding, predominant breastfeeding, complementary breastfeeding and breastfeeding) based on age group. The Fisher's and chi-square tests were used to investigate possible associations between the variables and breastfeeding.

\section{Results}

The prevalence of breastfeeding in children under six months of age was 37.3\%. Semi-solid foods were introduced abundantly during the infants' fifth month of age. In the sixth month, the diet of $50.7 \%$ of the children contained complementary foods in addition to breast milk. The prevalence of water, fruit and family food intakes among non-breastfed infants under six months of age was significantly higher that of their breastfed counterparts. There were no significant differences between exclusive breastfeeding in the first six months and the studied maternal variables.

\section{Conclusion}

Although the prevalence of exclusive breastfeeding in the first six months of an infant's life in Alfenas is higher than that of other cities, it still needs plenty of improvement to meet the recommendation that infants should be exclusively breastfed in that period.

Indexing terms: Breast feeding. Milk, human. Infant nutrition. Prevalence.

\section{N T R O D U ÇÃ O}

O leite humano é indiscutivelmente o alimento que reúne as características nutricionais ideais para a criança nos seis primeiros meses de vida. Além de proporcionar vantagens imunobiológicas e psicológicas, o leite materno também é fundamental na prevenção da morbimortalidade infantil1.

São inúmeras as pesquisas que fundamentam que, até os seis meses de idade, o leite materno é capaz de suprir as necessidades da criança, não havendo necessidade de qualquer outro líquido, como água, chá ou suco. Conforme estudos, após esse período, é importante a alimentação complementar oportuna e adequada quanto à frequência, consistência, aporte calórico e quantidade de micronutrientes, como ferro, zinco, cálcio, vitamina $\mathrm{A}$, vitamina $\mathrm{C}$ e folatos ${ }^{2}$. Contudo, o início da alimentação complementar tem ocorrido de forma inadequada, o que pode repercutir negativamente sobre a saúde da criança ${ }^{3}$.

Quando alimentos complementares são introduzidos precocemente, além de haver uma redução no tempo de aleitamento, há também uma interferência na absorção de nutrientes importantes presentes no leite materno, como ferro e zinco ${ }^{4}$.

Apesar de, a partir dos anos noventa, ter-se observado aumento dos índices de amamentação no mundo, 36\% dos recém-nascidos rece- 
bem Aleitamento Materno Exclusivo (AME) durante os primeiros seis meses de vida, e as práticas inadequadas de alimentação complementar ainda são generalizadas 5 .

No Brasil, a situação é semelhante, pois, mesmo tendo observada a tendência crescente da prática do Aleitamento Materno (AM) a partir do último quarto do Século $X X$, muitas crianças ainda deixam de recebê-lo durante os primeiros meses de vida ${ }^{6}$. Neste século, a Pesquisa Nacional de Demografia e Saúde (PNDS) ${ }^{7}$ apontou a seguinte situação da amamentação em crianças menores de seis meses: não amamentadas (8,2\%); AME (39,8\%); Amamentação Predominante (AMP) (13,6\%); Amamentação Complementada (AMC) (52,0\%) e Amamentação (AM) (91,8\%).

Os resultados da II Pesquisa de Prevalência do Aleitamento Materno (PPAM) nas capitais brasileiras e Distrito Federal, divulgados pelo Ministério da Saúde ${ }^{8}$, mostraram que o tempo médio do período de aleitamento materno no País aumentou um mês e meio: passou de 296 dias, em 1999, para 342 dias, em 2008. O estudo revelou um aumento do índice de AME em crianças menores de quatro meses, que em 1999 era de $35 \%$, e passou para $52 \%$ em 2008 . Evidenciou, também, a prevalência de $41 \%$ de AME em menores de seis meses no conjunto das capitais brasileiras e Distrito Federal ${ }^{8}$, resultado muito semeIhante ao obtido na PNDS-20067.

Apesar de a prevalência da amamentação no Brasil ter crescido nas últimas décadas, ainda há muito que se realizar para que se possa atingir a recomendação da Organização Mundial da Saúde (OMS) ${ }^{9}$, de AME nos primeiros seis meses de vida da criança e manutenção da amamentação até o segundo ano de vida ou mais. Há necessidade de se monitorarem constantemente os indicadores de aleitamento materno, buscando determinantes que possam ser modificados, delineando novas intervenções e realizando novas pesquisas ${ }^{10}$.

Os indicadores de AM têm apresentado um comportamento heterogêneo entre as dife- rentes regiões e municípios brasileiros ${ }^{7,8,10-18}$. A II PPAM $^{8}$ mostrou que em 2008, as capitais de estados da região norte foram as que apresentaram maior prevalência de AME em menores de seis meses (45,9\%), e as da Região Nordeste, a pior situação (37,0\%). Entre as capitais, esse indicador variou de 27,1\% em Cuiabá (MT) a 56,1\% em Belém (PA), passando pelos 37,9\% apresentados por Belo Horizonte (MG).

A variação da prevalência da amamentação nas diferentes localidades é muito relevante do ponto de vista da gestão da atenção à saúde, pois é necessária a obtenção de dados confiáveis em uma determinada população, para que se possam implementar ações e políticas públicas de promoção, proteção e apoio à amamentação, específicas para as situações encontradas.

Até a proposição do presente estudo, o município de Alfenas (MG) não possuía estudos divulgados sobre a prevalência da amamentação no âmbito municipal. Considerando a relevância que índices confiáveis de aleitamento materno possuem para a melhoria da programação de ações efetivas voltadas a sua promoção, procurou-se determinar a prevalência da amamentação e avaliar o consumo de outros alimentos em crianças menores de seis meses, buscando associar o aleitamento materno com variáveis relacionadas à mãe e à criança.

\section{MÉ TODOS}

O município de Alfenas está situado na Região Sul do estado de Minas Gerais, Brasil, e possui uma população de 71628 habitantes, estimada pelo Instituto Brasileiro de Geografia e Estatística $(\mathrm{IBGE})^{19}$, em 2007. Nesse ano, nasceram vivas 1015 crianças, de mães residentes na área urbana e rural do município ${ }^{20}$.

Foi realizado estudo transversal, por meio de inquérito domiciliar, no qual foram entrevistadas pessoas responsáveis pelas crianças menores de seis meses, principalmente mães $(75,4 \%)$. A coleta foi realizada no período de 23 a 30 de junho de 2008, por duas pesquisadoras previamente 
treinadas. Foi utilizado um formulário contendo o recordatório de 24 horas da alimentação da criança e outras questões referentes à mãe e à criança. $\mathrm{O}$ instrumento de coleta de dados foi validado por meio de teste-piloto.

A população de estudo constituiu-se de 335 crianças menores de seis meses de idade, nascidas a termo (com idade gestacional entre 37 e 42 semanas) no período compreendido entre janeiro e maio de 2008, residentes na zona urbana do município de Alfenas. Definiu-se como critério de exclusão: ter nascido de gestação pré ou pós-termo, por entender que tal condição poderia interferir no resultado, diminuindo o tempo de amamentação por razões relacionadas à saúde da criança; e residir na zona rural, por dificuldade de acesso das entrevistadoras. Os dados de identificação e endereço das mães das crianças que compõem a população de estudo foram obtidos a partir da declaração de nascidos vivos, por meio do Sistema de Informações de Nascidos-Vivos (SINASC) e fornecidos pelo setor de Vigilância Epidemiológica da Secretaria Municipal do Desenvolvimento Social, Saúde e Meio Ambiente de Alfenas.

Foi realizado um processo de amostragem em múltiplo estágio, sendo o primeiro estratificado pelas diferentes regiões de residência da mãe no município, e o segundo estágio estratificado pelo mês de nascimento da criança. $O$ tamanho da amostra foi calculado utilizando-se a expressão dada por Thompson ${ }^{21}$ e, após, foi selecionada uma amostra de 150 crianças, por sorteio, adotando-se um nível de confiança de $95 \%$ e uma margem de erro desejada de $4 \%$. No período da coleta dos dados, procedeu-se a novo sorteio para substituir 24 crianças cujas mães (ou pessoas aptas ao fornecimento de informação confiável) não foram encontradas após duas tentativas de entrevista.

Os dados foram digitados em banco de dados criado no Programa Epi Info, versão $6.04^{22}$. Para análise dos dados, o Teste de Fischer e o Teste qui-quadrado foram empregados na avaliação dos fatores associados com o aleitamento ma- terno até o sexto mês, admitindo-se um nível de significância de 5\%. Foi avaliado, também, o grau de associação entre as diferentes categorias de amamentação e a idade da criança, calculando-se o coeficiente de correlação linear $(r)^{23}$. No cálculo da prevalência do aleitamento materno, foram utilizadas as categorias definidas pela Organização Mundial de Saúde ${ }^{24}$ :

- Aleitamento materno exclusivo: crianças que recebem somente leite materno, sem outros sólidos ou líquidos, com exceção de gotas ou xaropes contendo vitaminas, suplementos minerais ou medicamentos;

- Amamentação predominante: crianças que recebem leite materno como fonte predominante de nutrição, associado a água ou bebidas à base de água (água adocicada, chás, infusões), sucos de frutas, solução de sais de reidratação oral, porém, sem outro leite ou outros alimentos;

- Amamentação complementada: crianças que recebem leite materno e outros alimentos sólidos, semissólidos ou líquidos, incluindo leites não humanos.

- Aleitamento materno: crianças que recebem leite materno, independentemente de receber outro alimento ou leite artificial.

O estudo foi aprovado pelo Comitê de Ética e Pesquisa com Seres Humanos da UNIFAL (MG) (Protocolo no 23087.001117/2008-69), e todos os participantes assinaram um Termo de Consentimento livre e esclarecido antes de sua inclusão na amostra.

\section{RESULTADOS}

Trabalhou-se com uma amostra válida de 126 crianças, pois foram descartados dados referentes a 24 entrevistas, por terem sido realizadas em período posterior ao estipulado para a coleta. Em razão desse procedimento, não se obtiveram dados referentes a crianças menores de um mês, o que não comprometeu a análise, tendo em vista que estudos atuais mostram que, no Brasil, a grande maioria das crianças encontra-se em AM no primeiro mês de vida ${ }^{6,25}$. 
A maior parte das crianças estudadas foi do sexo masculino (54,8\%). Quanto ao local de nascimento, 116 (92,1\%) nasceram em dois hospitais conveniados com o Sistema Único de Saúde (SUS), enquanto somente 10 (7,9\%) nasceram em um hospital particular. Os resultados mostraram que $23,0 \%$ (29) das crianças não passavam por consulta periódica de puericultura. Relativamente a estas, $27(93,1 \%)$ respondentes relataram procurar assistência à saúde apenas quando a criança apresenta febre ou outra anormalidade. Das 97 crianças que realizavam puericultura, 42 (43,3\%) eram assistidas por serviços privados, 41 (42,3\%) frequentavam unidades de saúde da família e 14 $(14,4 \%)$ recebiam atendimento pediátrico em ambulatórios da Secretaria Municipal de Saúde.

A maioria das mães possuía idade entre 19 e 30 anos (66,7\%), tinha dois ou mais filhos (52,4\%), possuía vínculo conjugal $(76,2 \%)$ e não trabalhava fora do lar $(61,1 \%)$.

A situação das diferentes categorias de amamentação utilizadas pelas crianças por faixa etária é apresentada na Tabela 1. De todas as crianças que participaram do estudo, 97 (77,0\%) recebiam leite materno, sendo que $37,3 \%$ estavam em AME e $23,0 \%$ em AMP.

Analisando-se a correlação linear entre faixa etária e o percentual de crianças nas diferentes categorias de amamentação, verificou-se uma relação negativa para AME $(r=-0,962)$, AMP $(r=-0,9779)$ e AM $(r=-0,962)$, ou seja, com o aumento da idade, o percentual de crianças nessas categorias de aleitamento progressivamente dimi- nui. Já para AMC ( $r=0,9307)$, a relação foi positiva, pois o percentual foi aumentando com o aumento da idade das crianças.

Observou-se uma tendência decrescente do AM que se inicia no terceiro mês de vida da criança e chega ao sexto mês com $65,0 \%$ das crianças recebendo leite materno. A tendência à queda é bem mais acentuada quando se analisa a proporção de crianças em AME, com um percentual de $63,6 \%$ no segundo mês, e apenas $7,7 \%$ no sexto. A queda mais intensa foi observada do quinto para o sexto mês. Simultaneamente à diminuição da AME há também uma queda da prevalência da AMP, pois alimentos semissólidos e outros leites passaram a ser introduzidos na alimentação, aumentando a proporção de crianças em amamentação complementada.

A introdução de alimentos semissólidos ocorreu mais acentuadamente a partir do quinto mês de vida. Verificou-se que, no quarto mês de vida, $11,3 \%$ das crianças recebiam outros tipos de alimentos, enquanto no sexto mês já se encontravam em AMC 50,7\% delas. A utilização de sucos, frutas e papas salgados (feijão e/ou legumes e/ou carnes) na alimentação das crianças ocorreu a partir do terceiro mês de vida, aumentando a frequência com o aumento da idade. No sexto mês, 38,6\% das crianças recebiam suco, $42,3 \%$ consumiam frutas e $50,0 \%$ já estavam sendo alimentadas com papas salgadas.

A introdução de leite não materno ocorreu precocemente a partir do terceiro mês de vida, mantendo tendência crescente até o quinto mês,

Tabela 1. Prevalência das diferentes categorias de amamentação por faixa etária em crianças até o sexto mês de idade. Alfenas (MG), 2008.

\begin{tabular}{|c|c|c|c|c|c|c|c|c|c|}
\hline \multirow{2}{*}{ Faixa etária (meses) } & \multirow{2}{*}{ Total crianças } & \multicolumn{2}{|c|}{ AME } & \multicolumn{2}{|c|}{ AMP } & \multicolumn{2}{|c|}{ AMC } & \multicolumn{2}{|c|}{ AM } \\
\hline & & $n$ & $\%$ & $n$ & $\%$ & $n$ & $\%$ & $n$ & $\%$ \\
\hline $1 \vdash 2$ & 11 & 7 & 63,6 & 4 & 36,4 & - & - & 11 & 100,0 \\
\hline $2 \vdash 3$ & 33 & 16 & 48,5 & 9 & 27,3 & 2 & 6,1 & 27 & 81,8 \\
\hline $3 \vdash 4$ & 28 & 13 & 46,4 & 7 & 25,0 & 2 & 7,1 & 22 & 78,6 \\
\hline $4-5$ & 28 & 9 & 32,1 & 5 & 17,8 & 6 & 21,4 & 20 & 71,4 \\
\hline $5 \vdash 6$ & 26 & 2 & 7,7 & 4 & 15,4 & 11 & 42,3 & 17 & 65,4 \\
\hline Total $<6$ & 126 & 47 & 37,3 & 29 & 23,0 & 21 & 16,7 & 97 & 77,0 \\
\hline
\end{tabular}

AME: aleitamento materno exclusivo; AMP: amamentação predominante; AMC: amamentação complementada; AM: aleitamento materno. 
e diminuindo no sexto mês. Das 39 crianças que recebiam leite não materno, 13 (33,3\%) faziam uso de fórmulas lácteas infantis e $26(66,7 \%)$ recebiam leite fluido de origem animal, sendo que 11 $(42,3 \%)$ recebiam leite in natura adquirido no comércio informal.

As crianças menores de seis meses não amamentadas, comparadas com as amamentadas, apresentaram prevalências significativamente maiores $(p<0,05)$ quanto ao consumo de água, frutas e comida da família (Tabela 2). Quanto ao consumo de sucos, chás e papas salgadas e à utilização de chupetas, não foram observadas diferenças estatisticamente significativas entre crianças amamentadas e não amamentadas (Tabela 2).

Tabela 2. Consumo de água, sucos, chás, frutas, papas salgadas, comida da família e uso de chupeta, conforme a presença ou ausência de aleitamento materno em crianças menores de 6 meses de idade. Alfenas (MG), 2008.

\begin{tabular}{|c|c|c|c|}
\hline \multirow{3}{*}{ Variáveis } & \multicolumn{2}{|c|}{ Aleitamento materno } & \multirow{3}{*}{ Valor de $p$} \\
\hline & Sim & Não & \\
\hline & $\%$ & $\mathrm{n}$ & \\
\hline
\end{tabular}

\begin{tabular}{|c|c|c|c|c|c|}
\hline \multicolumn{6}{|c|}{ Água } \\
\hline Sim & 36 & 37,1 & 23 & 79,3 & 0,0001 \\
\hline Não & 61 & 62,9 & 6 & 20,7 & \\
\hline \multicolumn{6}{|c|}{ Sucos } \\
\hline Sim & 15 & 15,5 & 6 & 20,7 & 0,5716 \\
\hline Não & 82 & 84,5 & 23 & 79,3 & \\
\hline \multicolumn{6}{|c|}{ Chás } \\
\hline Sim & 9 & 9,3 & 3 & 10,3 & 0,9940 \\
\hline Não & 88 & 90,7 & 26 & 89,7 & \\
\hline \multicolumn{6}{|c|}{ Frutas } \\
\hline Sim & 11 & 11,3 & 9 & 31,0 & 0,0186 \\
\hline Não & 86 & 88,7 & 20 & 69,0 & \\
\hline \multicolumn{6}{|c|}{ Papas salgadas ${ }^{*}$} \\
\hline Sim & 15 & 15,5 & 8 & 27,6 & 0,1711 \\
\hline Não & 82 & 84,5 & 21 & 72,4 & \\
\hline \multicolumn{6}{|c|}{ Comida família } \\
\hline Sim & - & - & 4 & 13,8 & 0,0024 \\
\hline Não & 97 & 100,0 & 25 & 86,2 & \\
\hline \multicolumn{6}{|c|}{ Uso de chupeta } \\
\hline Sim & 53 & 54,6 & 21 & 72,4 & 0,1316 \\
\hline Não & 44 & 45,4 & 8 & 27,6 & \\
\hline
\end{tabular}

* Papas elaboradas com legumes e/ou feijão e/ou carnes.
Com relação ao uso de chupetas, apesar de não ter sido encontrada associação significativa com a interrupção da amamentação, foi observado um percentual elevado $(60,0 \%)$ de crianças menores de seis meses, amamentadas e não amamentadas, que as utilizavam.

Não foi encontrada diferença estatisticamente significativa $(p>0,05)$ na associação entre amamentação exclusiva até o sexto mês e as variáveis maternas: situação conjugal, paridade, grau de instrução e ocupação.

\section{DIS C U S S Ã O}

Em comparação com os resultados obtidos em estudos realizados na década de 2000 , a prevalência da $A M$ de $77,0 \%$ em menores de seis meses foi inferior à descrita em Botucatu-SP referente ao ano de $2004^{15}$, e à atingida em Joinville $(\mathrm{SC})^{16}$ em 2005 , respectivamente $79,2 \%$ e $84,2 \%$. Destaca-se ainda que esse resultado foi muito inferior aos 91,8\% encontrados para o Brasil na PNDS-2006 7 .

Com relação à $\mathrm{AME}$, o município de Alfenas apresentou para crianças menores de seis meses o percentual de $37,3 \%$, resultado melhor que o de Botucatu $(29,6 \%)^{15}$, que o de Londrina (PR) em $2002(21,0 \%)^{14}$, que o de Bauru (SP) em $2006(24,2 \%)^{10}$, e bem maior do que os $15,7 \%$ obtidos em Juiz de Fora (MG) ${ }^{17}$ em 2002. Porém, o resultado deste estudo foi inferior ao encontrado em Joinville $(43,6 \%)^{16}$, ao obtido em 2006 para o Brasil $(39,8 \%)^{7}$, e aos $41,0 \%$ encontrados nas capitais brasileiras na IIPPAM ${ }^{8}$ realizada em 2008.

Apesar de Alfenas possuir alguns indicadores de AM superiores aos de outros municípios, a situação ainda é preocupante principalmente com relação à prevalência de 37,3\% de AME em menores de seis meses, pois, de acordo com parâmetros estabelecidos pela OMS, a faixa de 12,0\% a 49,0\%, para esse indicador é considerada ruim ${ }^{8}$.

O estudo constatou a introdução precoce de alimentação complementar, tanto alimentos 
semissólidos quanto outros leites que não o materno, o que também foi evidenciado em estudo realizado em 2002 na região do Alto Jequitinhonha em Minas Gerais ${ }^{18}$, e também nas capitais brasileiras e Distrito Federal em 2008 ${ }^{8}$. A principal contraindicação à introdução precoce de alimentos complementares é sua relação direta com o aumento da morbimortalidade, pois, além de serem considerados potenciais fontes de contaminação, eles fazem diminuir a ingestão de leite materno e, assim, a criança recebe menos fatores de proteção ${ }^{26}$. Vale ressaltar que a preocupação com a morbimortalidade se potencializa, neste estudo, quando se observa que 11 crianças recebiam leite in natura, sem passar por qualquer processo de pasteurização ou outro, fora do âmbito doméstico, que o tornasse próprio para o consumo. Outro fator importante a ser considerado na introdução precoce de alimentos semissólidos é a imaturidade neurológica existente antes do sexto mês de vida da criança para a deglutição de alimentos não líquidos, ou seja, crianças dessa faixa etária não estão fisiologicamente preparadas para digerir alimentos sólidos que Ihes são oferecidos ${ }^{27}$. O fato de a introdução de alimentos semissólidos ter ocorrido de forma mais acentuada aos quatro meses de vida da criança pode estar relacionado ao término da licença-maternidade e ao retorno das mães ao trabalho ${ }^{28}$.

A maior prevalência significativa do consumo de água, frutas e comida da família, encontrada nas crianças menores de seis meses não amamentadas, também foi observada no estudo de Vieira et al. ${ }^{28}$ com crianças menores de cinco meses. Por outro lado, no que se refere ao consumo de sucos, chás e papas e ao uso de chupetas, os resultados desta pesquisa, embora tenham apresentado maior prevalência entre as crianças não amamentadas, não mostram associação estatística significativa como aquela encontrada no estudo acima mencionado ${ }^{28}$.

Apesar de não ter havido resultado significativo entre uso de chupeta e desmame, foi observada uma elevada frequência da utilização de chupetas entre as crianças menores de seis meses em Alfenas $(60,0 \%)$, sendo superior à apresentada em Bauru em $2006(54,0 \%)^{10}$. A interferência negativa do uso de chupetas na amamentação tem sido apontada por vários estudos ${ }^{10,29-31}$. Porém, o consenso existente sobre tal interferência foi questionado pelo estudo de O'Connor et al. ${ }^{32}$, que concluiu não haver evidências científicas suficientes para atribuir o desmame ao uso de chupetas. O impasse sobre desaconselhar ou não o uso de chupetas iniciou-se após a recomendação da Academia Americana de Pediatria, em 2005, de utilizá-las em bebês para a redução da síndrome da morte súbita no primeiro ano de vida ${ }^{32}$. O trabalho citado concluiu, também, que há necessidade de se realizarem outros estudos qualitativos e quantitativos para um melhor entendimento sobre a questão, pois a associação entre uso de chupetas e menor duração da amamentação, encontrada nos estudos observacionais, provavelmente reflete vários outros fatores, como dificuldades na prática de amamentar ou decisão de desmamar ${ }^{32}$. A não recomendação do uso de chupetas realizada por muitos profissionais e serviços de saúde no Brasil parece estar refletindo na diminuição dessa prática, pois a II PPAM ${ }^{8}$ mostrou que, de 1999 para 2008 , houve uma redução expressiva $(15,1 \%)$ de sua utilização em menores de 12 meses, no conjunto das capitais brasileiras.

Diferentemente dos resultados obtidos em outras pesquisas ${ }^{8,13,33}$, não foi encontrada diferença estatisticamente significativa entre a amamentação exclusiva até o $6^{\circ}$ mês e as seguintes variáveis maternas: situação conjugal, paridade, grau de instrução e ocupação $(p>0,05)$.

Cabe ressaltar que quase um quarto das crianças menores de seis meses não realizava atendimento de puericultura, evidenciando que é preciso melhorar a cobertura da atenção básica à saúde/saúde da família no município.

\section{O N CLUS Ã O}

Apesar de vários resultados se apresentarem comparativamente superiores aos de outros 
municípios, a prevalência do aleitamento materno em Alfenas ainda está distante do preconizado pelo Ministério da Saúde e pela Organização Mundial de Saúde. É possível afirmar que existe uma margem para a melhoria da qualidade nos serviços básicos existentes, no que tange às ações de promoção, proteção e apoio à amamentação.

Não foram encontrados parâmetros anteriores que possibilitassem comparar os resultados deste estudo e avaliar a tendência do aleitamento materno no decorrer dos anos em Alfenas. Indica-se que sejam realizados outros estudos que avaliem a situação da amamentação e dos hábitos alimentares de crianças, de forma a propiciar o monitoramento da tendência das práticas alimentares na infância e a subsidiar políticas, programas e ações de promoção, proteção e apoio ao aleitamento materno no município.

\section{A GRADECIMENTOS}

Aos funcionários da Vigilância Epidemiológica da Secretaria Municipal do Desenvolvimento Social, Saúde e Meio Ambiente de Alfenas pelo auxílio no acesso aos dados do SINASC.

\section{COLABORADORES}

C.I.S. ARANTES participou da concepção, do desenho, da análise, de interpretação e da elaboração do artigo. M.M. OLIVEIRA participou do desenho, coleta e análise dos dados e elaboração do artigo. T.C.R. VIEIRA participou do desenho, da coleta e de análise dos dados. L.A. BEIJO, C.V.C. GRADIM e S.L.T. GOYATÁ participaram da análise e de interpretação dos resultados.

\section{REFERÊ NCIAS}

1. Bueno LGS, Teruya KM. Aconselhamento em amamentação e sua prática. J Pediatr. 2004; 80(5 Supl.): 126-30.

2. Brasil. Ministério da Saúde. Organização Pan-Americana da Saúde. Guia alimentar para crianças menores de 2 anos de idade. Brasília: Ministério da Saúde; 2002. Série A. Normas e Manuais Técnicos.
3. Saldiva SRDM, Escuder MM, Mondini L, Levy RB, Venâncio SI. Práticas alimentares de crianças de 6 a 12 meses e fatores maternos associados. J Pediatr. 2007; 83(1):53-8.

4. Monte CMG, Giugliani ERJ. Recomendações para alimentação complementar da criança em aleitamento materno. J Pediatr. 2004; 80(5 Supl.):131-42.

5. Sguassero Y. Duración óptima de la lactancia materna exclusiva: comentario de la Biblioteca de Salud Reproductiva de la OMS. Ginebra: OMS; 2008 [acceso: 2009 dez 8]. Disponible en: <http:// apps.who.int/rhl/pregnancy_childbirth/care_after_ childbirth/yscom/es/>.

6. Sena MCF, Silva EF, Pereira MG. Tendências do aleitamento materno no Brasil no último quarto do século XX. Rev Bras Epidemiol. 2007; 10(4):499-505.

7. Segall-Corrêa AM, Marin-León L. Amamentação e alimentação. In: Brasil. Ministério da Saúde. PNDS 2006: Pesquisa Nacional de Demografia e Saúde da Criança da Mulher [Relatório]. Brasília: Ministério da Saúde; 2008 [acesso 23 nov 2009]. Disponível em: <http://bvsms.saude.gov.br/bvs/publi cacoes/pnds_crianca_mulher.pdf>.

8. Brasil. Ministério da Saúde. Secretaria de Atenção à Saúde. II Pesquisa de prevalência de aleitamento materno nas capitais brasileiras e Distrito Federal [Internet]. Brasília: Ministério da Saúde; 2009 [acesso: 2009 dez 1]. Disponível em: <http:// bvsms.saude.gov.br/bvs/publicacoes/pesquisa_ prevalencia_aleita mento_materno.pdf>.

9. World Health Organization. The optimal duration of exclusive breastfeeding a systematic review. 2002. [cited 2009 Feb 11]. Available from: <http:// www.who.int>.

10. Parizoto GM, Parada CMGL, Venâncio SI, CarvaIhaes MABL. Tendência e determinantes do aleitamento materno exclusivo em crianças menores de 6 meses. J Pediatr. 2009; 85(3):201-8.

11. Passos MC, Lamounier JA, Silva CAM, Freitas SN, Baudson MFR. Práticas de amamentação no município de Ouro Preto, MG, Brasil. Rev Saúde Pública. 2000; 34(6):617-22.

12. Montrone VG, Arantes CIS. Prevalência do aleitamento materno na cidade de São Carlos. J Pediatr. 2000; 76(2):138-42.

13. Vituri SC, Brito ASJ. Prevalência do aleitamento materno em crianças até o sexto mês de idade na cidade de Maringá, estado do Paraná, Brasil. Acta Sci Health Sci. 2003; 25(2):141-6.

14. Vannuchi MTO, Thomson Z, Escuder MML, Tacla MTGM, Vezozzo KMK, Castro LMCP, et al. Perfil do aleitamento materno em menores de um ano no município de Londrina, Paraná. Rev Bras Saúde Mater Infant. 2005; 5(2):155-62. 
15. Ferreira L, Parada CM, Carvalhaes MA. Tendências do aleitamento materno em município da região centro-sul do estado de São Paulo: 1995-19992004. Rev Nutr [Internet]. 2007 [acesso 2009 jun 10]; 20(3):265-73. Disponível em: <http://www. scielo.br/pdf/rn/v20n3/05.pdf>. doi: 10.15 90/S14 15-52732007000300005.

16. Franco SC, Nascimento MBR, Reis MAM, Issler H, Grisi SJFE. Aleitamento materno exclusivo em lactentes atendidos na rede pública do município de Joinville, Santa Catarina, Brasil. Rev Bras Saúde Matern Infant. 2008; 8(3):291-7.

17. Afonso VW, Ribeiro LC, Alves MJM, Teixeira MTB, Dain S. Prevalência do aleitamento materno em município de médio porte do sudeste brasileiro. Rev APS. 2008; 11(4):406-12.

18. Silveira FJF, Lamounier JA. Prevalência do aleitamento materno e práticas de alimentação complementar em crianças com até 24 meses de idade na região do Alto Jequitinhonha, Minas Gerais. Rev Nutr [Internet]. 2004 [acesso 2009 jun 10]; 17(4): 437-47. Disponível em: <http://www.scielo.br/pdf/ rn/v17n4/22892.pdf>. doi: 10.1590/S141552732 004000400004.

19. Brasil. Ministério do Planejamento, Orçamento e Gestão [Internet]. Brasília: Ministério do Planejamento, Orçamento e Gestão; 2007 [acesso 2008 set 20]. Dispo-nível em: <http://www.ibge.gov.br>.

20. Brasil. Ministério da Saúde. Secretaria de Vigilância em Saúde. Sistema de informações sobre nascidos-vivos [Internet]. Brasília: 2007 [acesso 2009 dez 1]. Disponível em: <http://tabnet.datasus.gov.br/ cgi/tabcgi.exe?sinasc/cnv/nvmg. def>.

21. Thompson S K. Sampling. New York: John Wiley and Sons; 1992.

22. Dean AG, Dean JA, Coulombier D, Brendel KA, SmithDC, Burton AH, et al. Epi Info, version 6. Atlanta: Centers for Disease Control and Prevention; 1996.

23. Bussab O, Morettin PA. Estatística básica. $5^{a}$ ed. São Paulo: Saraiva; 2002.
24. World Health Organization. Indicators for assessing breastfeeding practices. Geneva: WHO; 1991.

25. Sena MCF, Silva EF, Pereira MG. Prevalência do aleitamento materno nas capitais brasileiras. Rev Assoc Med Bras. 2007; 53(6):520-4.

26. Giugliani ERJ, Victora CG. Alimentação complementar. J Pediatr. 2000; 76(3): 253-62.

27. World Health Organization. Complementary feeding of young children in developing countries: a review of current scientific knowledge. Geneva: WHO; 1998.

28. Vieira GO, Silva LR, Vieira TO, Almeida JAG, Cabral VA. Hábitos alimentares de crianças menores de 1 ano amamentadas e não amamentadas. J Pediatr. 2004; 80(5):411-6.

29. Contrim LC, Venâncio SI, Escuder MM. Uso de chupeta e amamentação em crianças menores de quatro meses no estado de São Paulo. Rev Bras Saude Mater Infant. 2002; 2(3):245-52.

30. Lamounier JA. O efeito de bicos e chupetas no aleitamento materno. J Pediatr. 2003; 79(4):284-6.

31. Soares MEM, Giugliani ERJ, Braun ML, Salgado ACN, Oliveira AP, Aguiar PR. Uso de chupeta e sua relação com o desmame precoce em população de crianças nascidas em Hospital Amigo da Criança. J Pediatr. 2003; 79(4):309-16.

32. O'Connor NR, Tanabe KO, Siadaty MS, Hauck FR. Pacifiers and breastfeeding: a systematic review. Arch Pediatr. Adolesc Med [Internet]. 2009 [cited 2009 Jun. 25]; 163(4). Available from: <http//www. archpediatrics.com at Capes Consortia>.

33. Carrascoza KM, Costa Júnior AL, Moraes ABA. Fatores que influenciam o desmame precoce e a extensão do aleitamento materno. Estud Psicol. 2005; 22(4):433-40. doi: 10.1590/S0103-166 X2005000400011.

Recebido em: 30/7/2009

Versão final reapresentada em: 1/7/2010 Aprovado em: 15/7/2010 
